Relative Consumption Concerns and the Optimal Tax Mix

by

Paul Eckerstorfer

Working Paper No. 1114

October, 2011

Johannes Kepler University of Linz Department of Economics Altenberger Strasse 69 A-4040 Linz - Auhof, Austria www.econ.jku.at 


\title{
Relative Consumption Concerns and the Optimal Tax Mix
}

\author{
by \\ Paul Eckerstorfer* \\ November 14, 2012
}

\begin{abstract}
This articles studies the optimal tax mix (taxes on income and commodities) under asymmetric information in a two-type model, when individuals make relative consumption comparisons. The model includes both positional and nonpositional goods, taking into account the fact that relative concerns matter for some but not for all commodities. We find that in general the whole tax system is affected by the externalities caused by the consumption of positional goods, notably also the taxes on income and on a nonpositional good. The tax rates on positional goods are higher than in the absence of status effects, reflecting their Pigouvian role. The sign of the Pigouvian part in the income tax schedule is ambiguous and depends crucially on whether status goods are complements or substitutes to leisure.
\end{abstract}

Keywords: Optimal Taxation, Externalities, Relative Consumption

JEL Classification: D82, H21, H24, H26

*Department of Economics, Altenbergerstr. 69, 4040 Linz, Austria.

E-mail: paul.eckerstorfer@jku.at 


\section{Introduction}

This paper studies the optimal mix of a nonlinear income tax and proportional commodity taxes in the presence of relative consumption concerns. Most part of the optimal taxation literature assumes that the utility of individuals depends only on their own consumption of goods and leisure. However, there is increasing empirical evidence suggesting that individuals value not only their own absolute consumption but also their relative consumption with respect to others (for an overview see, e.g., Clark et al. 2008). Moreover, there is evidence indicating that some goods are more positional than others, that is relative concerns matter more for some commodities than for others (e.g. Alpizar et al. 2005 and Solnick and Hemenway 2005). Typically, visible forms of consumption such as clothing or housing tend to be more positional than less visible forms such as food or insurance consumption. In accordance with this empirical evidence we construct a model that allows us to analyze the optimal tax structure assuming that some but not all commodities are positional.

The idea that the quest for status is an important characteristic of human behavior has already been raised by ancient philosophers (see Aristotle 1924, Rhetoric (II), Plato 1967, The Republic (II)). ${ }^{1}$ In economics, already Adam Smith (1759) and later John Rae (1834) and Thorstein Veblen (1899) have emphasized the importance of relative concerns for individual well-being. ${ }^{2}$ Veblen (1899) coined the term 'conspicuous consumption' for the spending on goods which serve to display ones social status. The first one who formalized this idea was Duesenberry (1949) in his relative income hypothesis, where he emphasized the importance of relative concerns for consumption and saving decisions. Since the late 70's a literature on optimal policy issues when relative consumption matters, has gradually developed (e.g. Boskin and Sheshinski 1978, Oswald 1983, Ng 1987, Ireland 2001, Wendner and Goulder 2008, Aronsson and Johansson-Stenmann 2008, Micheletto 2010). ${ }^{3}$ It has become evident from these studies that several standard results concerning optimal tax policy and public good provision do not hold or at least have to be adapted if one takes status effects into account.

However, most part of this literature assumes that there is only one consumption good and thus, does not differentiate between positional and nonpositional forms of consumption, as we do. Further, it is typically assumed that individuals compare their own consumption to the average consumption in the economy. In this paper we model reference consumption levels in a more general way, which allows us to introduce substantial heterogeneity among individuals with respect to status effects. First, in our framework different types of individuals in general have different reference

\footnotetext{
${ }^{1}$ For example, in The Republic (II) (2008, p. 62) Plato wrote: "Since, then, as philosophers prove, appearance tyrannizes over truth and is lord of happiness, to appearance I must devote myself."

${ }^{2}$ In the Theory of Moral Sentiments Smith (1759, p. 181) wrote: "The poor man's son...when he begins to look around him, admires the condition of the rich. He finds the cottage of his father too small...It appears in his fancy like the life of some superior rank of beings, and, in order to arrive at it, he devotes himself for ever to the pursuit of wealth and greatness."

${ }^{3}$ Relative consumption concerns were also introduced in models analyzing growth (e.g. Corneo and Jeane 2001, Wendner 2010) or asset pricing (e.g. Dupor and Liu 2003).
} 
levels. Second, the consumption of different types might be weighted differently in the formation of the reference levels which allows us to model for the idea that there are high- and low status individuals. ${ }^{4}$ We claim that our flexible formulation of the reference level conforms more to what one observes in reality than taking the average consumption as the unique reference level for the whole population.

This paper is also related to the literature that studies optimal mixed taxation (income and commodities) in an asymmetric information setting a la Mirrlees (1971) in the presence of consumption externalities (Pirttilä and Tuomala 1997, Cremer et al. 1998, Kopczuk 2003, Micheletto 2008). Our paper is related to these studies because the consumption of status goods also causes an externality since when consuming such goods individuals impose a utility loss on others by worsening their relative position. One important result from these previous studies is that the so called additivity property first discovered by Sandmo (1975) also carries over to the more general optimal mixed tax case with heterogeneous agents, at least if the externality is of the atmospheric type. ${ }^{5}$ The additivity property states that an externality is best addressed by imposing a tax directly on the externality-generating good while the rest of the tax system should be unaffected by the externality. However, in a recent paper Micheletto (2008) has shown that for the additivity property to be valid it is essential that different types are equally effective as externality-generating units, i.e. it should not matter which individual increases the level of the externality with his/her consumption (as it is the case if the externality is of the atmospheric type). This is typically fulfilled for environmental externalities but as argued above, this needs not be the case for positional externalities. In accordance with Micheletto's result we show that in our model in general the whole tax system is affected by the positional externalities. Further, we extend previous work on optimal mixed taxation and consumption externalities to a multi-externality setting. So far the literature has confined the analysis to cases with only one externality-generating good. ${ }^{6}$ This extension to a setting with two positional goods allows to study the impact of potential interdependences between the positional goods on optimal tax policy.

The analysis is conducted in a two-group optimal tax model (Stiglitz 1982) where individuals differ in earning abilities and tastes. Taste differences are reflected by different reference levels for the two types. As is common in the optimal taxation literature, earning abilities are private information of the individual. Hence, firstbest taxation of abilities is not feasible which is why the government has to use a general income tax as a second-best instrument. There are three consumption goods in the economy. Two of them are positional goods and one good is a nonpositional good, where only absolute consumption matters. For simplicity we assume a linear

\footnotetext{
${ }^{4}$ In Wendner (2012) the consumption of different individuals is also allowed to be weighted differently in the formation of the reference level, but in his framework all individuals have the same reference level.

${ }^{5}$ The term atmospheric externality was introduced by Meade (1952). It is used when the externality depends on the total consumption of a particular good.

${ }^{6}$ An exception is Eckerstorfer (2012) who studies the optimal income and commodity tax structure in a multi-externality model, where however, in contrast to the present study, the externalities are restricted to be of the atmospheric type.
} 
production technology with labor as the only input, which allows us to focus on the consumption side of the economy. Each commodity is subject to a proportional tax rate, i.e. the tax system consists of a nonlinear income tax and proportional commodity tax rates.

We show that in general all available tax instruments, including the tax on the nonpositional good and the income tax, are required to internalize the positional externalities which violates the additivity property. The intuition for this result is that if at the margin the status consumption of some individuals is socially more harmful than the same consumption of others, it would be desirable to tax status consumption of these individuals at different rates. Since commodity taxes are restricted to be proportional this is not feasible and the government has to exploit other ways to channel individual consumption decisions in the desired direction. However, we show that this result hinges crucially on the structure of compensated prices affects and we provide conditions which are sufficient to restore the additivity property for the commodity tax structure (not for the income tax) even if the consumption of the two types is weighted differently in the formation of the reference level. Such conditions do not exist for the income tax which contains Pigouvian elements as soon as the consumption of the two types is weighted differently. An interesting implication of our multi-externality assumption is the result that the Pigouvian elements in the tax formulas depend on both externalities, implying that the interdependence between the positional commodities is of importance.

We also discuss the optimal income tax schedule if commodity taxes are restricted to be uniform across commodities for example due to political economy reasons. In that case only the income tax can be used to correct for the externalities. An implication of our assumption that there are both positional and nonpositional forms of consumption is that the sign of the Pigouvian part in the income tax schedule is ambiguous and depends crucially on whether the positional goods are complements or substitutes to leisure. Intuitively, if the status good is a complement to leisure (e.g. playing golf), a higher marginal income tax rate induces individuals to consume more leisure, and hence, to consume more of the status good. Clearly, the reduction in income has an opposite effect, which is why the overall effect is ambiguous. Moreover, we show that if the consumption of the rich is weighted higher in the formation of the reference levels (upward comparison) then with a mild additional assumption the Pigouvian part in the income tax schedule is progressive.

The rest of the paper is organized as follows. In Section 2 we present the model and the maximization problem of the households and the government. In Section 3 we discuss the optimal commodity tax structure and in Section 4 the results on the optimal income tax schedule. Section 5 provides some discussion of the results. Proofs are provided in the Appendix and the derivations of the results are provided in a technical online Appendix. 


\section{The model}

We consider an economy consisting of two types of individuals $i=L, H$, who differ in earning abilities $\omega_{L}<\omega_{H}$. The size of the population is normalized to one and $\pi_{i}$ represents the proportion of individuals of type $i$ in the population. By providing labor supply $l_{i}$ individuals earn gross income $z_{i}=\omega_{i} l_{i}$. Gross income $z_{i}$ is subject to a nonlinear income tax and the resulting net income is denoted by $x_{i}$. Individuals spend their net income on the consumption of three commodities $c_{j}, j=1,2,3$, which are produced with a linear technology with labor as the only input to production. Quantities are chosen in such a way that the (constant) marginal costs of production are equal to one, i.e. the producer prices of all commodities are equal to one.

\section{The consumers' problem and relative concerns}

In our model commodities $k=1,2$ are assumed to be positional goods, i.e. for these goods individuals care not only about their absolute value of consumption but also about their relative consumption with respect to others. ${ }^{7}$ Commodity 3 on the other hand is a nonpositional good where only absolute consumption matters. For the positional commodities each individual compares his/her own consumption to some reference consumption level which is determined for the $L$ type by

$$
\overline{c_{k L}}=\alpha_{k L}(L) \pi_{L} c_{k L}+\alpha_{k H}(L) \pi_{H} c_{k H}
$$

and for the $H$ type by

$$
\overline{c_{k H}}=\alpha_{k L}(H) \pi_{L} c_{k L}+\alpha_{k H}(H) \pi_{H} c_{k H}
$$

with $k=1,2 .{ }^{8}$ As usual reference consumption levels are treated as exogenous to the individual. The intuition for this assumption is that individuals consider their own contribution to the reference levels as extremely small.

From (1) and (2) it can be seen that we model the reference consumption level in a very flexible way. The nonnegative exogenous weights $\alpha_{k L}(i)$ and $\alpha_{k H}(i)$ denote by how much the consumption of one unit of good $k$ of individuals $L$ and $H$, respectively, raises the reference level of individual $i$ for good $k$. This formulation allows for the possibility that low- and high-able individuals have different reference levels. Further, the consumption of the two types is allowed to be weighted differently in the formation of the reference level $\left(\alpha_{k L}(i) \neq \alpha_{k H}(i)\right)$, i.e. the consumption of the $H$-types might be weighted higher (lower) in the formation of the reference levels than the same consumption of the $L$-types. In its simplest form where all weights are equal to one, reference consumption levels are equal to the average consumption of the specific com-

\footnotetext{
${ }^{7}$ We use the index $j=1,2,3$ when we refer to all commodities and the index $k=1,2$ when we refer to the positional commodities.

${ }^{8}$ In order to form the reference consumption levels as given by (1) and (2), individuals need to observe the consumption of at least one high- and one low-able individual, of whom they know the ability. Since the fractions $\pi_{i}$ are common knowledge, they need not identify the ability of each individual.
} 
modity. In that case both types face the same reference levels and their consumption is weighted equally in the formation of $\overline{c_{k i}}$. However, our formulation of the reference levels is also flexible enough to study other variants discussed in the literature, such as upward and within-group comparison, just by adapting weights properly. ${ }^{9}$

Both types have the same strictly concave utility function, $u\left(c_{1 i}, c_{2 i}, c_{3 i}, l_{i}, \overline{c_{1 i}}, \overline{c_{2 i}}\right)$, with first partial derivatives being positive with respect to $c_{1 i}, c_{2 i}, c_{3 i}$ and negative with respect to $l_{i}, \overline{c_{1 i}}, \overline{c_{2 i}} \cdot{ }^{10}$ We assume that $\partial\left(\left(\partial u_{i} / \partial l_{i}\right) /\left(\partial u_{i} / \partial c_{k i}\right)\right) / \partial \overline{c_{k i}}>0$, which defines a keeping up with the Joneses (KUJ) externality (see Dupor and Liu 2003). That is, an increase in the reference level for good $k$ increases the marginal utility of consuming good $k$ relative to that of leisure, and by this induces individuals to work more at given prices. Observe, that the consumption of good 1 and 2 gives rise to a negative externality, since individuals do not take into account the effect their own consumption of commodities 1 and 2 has on others via the reference levels (by worsening the relative position of others).

The individuals' maximization problem is broken into two steps. In a first step, an individual $i$ allocates a fixed amount of net income $x_{i}$ over the consumption goods. Consumer prices are denoted by $p_{j}=1+\tau_{j}, j=1,2,3$, i.e. the government imposes a proportional commodity tax $\tau_{j}$ on each of the three commodities. Maximizing utility subject to the private budget constraint yields conditional indirect utility for given gross and net income:

$$
v_{i}\left(x_{i}, z_{i}, p_{1}, p_{2}, p_{3}, \overline{c_{1 i}}, \overline{c_{2 i}}\right) \equiv \max _{c_{1 i}, c_{2 i}, c_{3 i}}\left\{u\left(c_{1 i}, c_{2 i}, c_{3 i}, z_{i} / \omega_{i}, \overline{c_{1 i}}, \overline{c_{2 i}}\right) \mid \sum_{j=1}^{3} p_{j} c_{j i} \leq x_{i}\right\} .
$$

Solving the private maximization problem described by (3) yields conditional demand functions

$$
c_{j i}=c_{j i}\left(x_{i}, z_{i}, p_{1}, p_{2}, p_{3}, \overline{c_{1 i}}, \overline{c_{2 i}}\right)
$$

$j=1,2,3$ and $i=L, H$. Observe that in general the demand for all commodities depends on the reference levels $\overline{c_{1 i}}$ and $\overline{c_{2 i}}$.

In a second step, individuals determine their optimal labor supply by maximizing conditional indirect utility $v_{i}\left(x_{i}, z_{i}, p_{1}, p_{2}, p_{3}, \overline{c_{1 i}}, \overline{c_{2 i}}\right)$ subject to the budget equation $x_{i}=z_{i}-T\left(z_{i}\right)$, where $T\left(z_{i}\right)$ denotes the nonlinear income tax function. Assuming that the income tax function is differentiable and letting the marginal income tax rate be denoted by $T^{\prime}\left(z_{i}\right)$, individuals choose their labor supply such that

$$
T^{\prime}\left(z_{i}\right)=1+\frac{\partial v_{i} / \partial z_{i}}{\partial v_{i} / \partial x_{i}}
$$

\footnotetext{
${ }^{9}$ Upward comparison means that individuals compare themselves to other individuals above them in the income distribution whereas within-group comparison means that individuals compare themselves to individuals in the same income group.

${ }^{10} \mathrm{As}$ in most other studies leisure is assumed to be nonpositional, which is justified by a number of empirical results (e.g. Solnick and Hemenway 1998, 2005). An exception is Aronsson and Johansson-Stenman (2012), who assume that individuals care about both relative consumption and relative leisure.
} 


\section{The government's problem}

The government's objective is to design a tax system that raises revenues and redistributes income in an efficient way and that takes into account the positional externalities induced by the consumption of goods 1 and 2. Since the government cannot observe the ability of individuals - only gross incomes and the distribution of types are observable - it cannot impose type specific first-best lump-sum taxes. It rather has to use a general income tax and commodity taxes as second-best instruments. The problem of finding an optimal income tax schedule can equivalently be stated by determining the optimal gross and net income bundles $x_{i}, z_{i}$ for each type (the optimal income tax is then implicitly determined by $\left.z_{i}-x_{i}, i=L, H\right)$. Commodity taxes are restricted to be proportional as consumption is assumed to be observable only in the aggregate, while individual consumption levels are private information. That is, the government does not know who buys how much of what good, which is why nonlinear commodity taxes are not feasible.

The utilitarian social welfare function, which is the objective function of the maximization problem of the government, reads

$$
\max _{\tau_{1}, \tau_{2}, \tau_{3}, x_{i}, z_{i}, \overline{c_{1 i}}, \overline{c_{2 i}}, i=L, H} f_{L} v_{L}\left(x_{L}, z_{L}, p_{1}, p_{2}, p_{3}, \overline{c_{1 L}}, \overline{c_{2 L}}\right)+f_{H} v_{H}\left(x_{H}, z_{H}, p_{1}, p_{2}, p_{3}, \overline{c_{1 H}}, \overline{c_{2 H}}\right),
$$

where $f_{L}$ and $f_{H}$, with $f_{L} \geq f_{H} \geq 0$, represent the weights of the two types of individuals including the fractions $\pi_{L}$ and $\pi_{H}$. We assume that the agent monotonicity condition is fulfilled, meaning that $M R S_{z x}^{L}>M R S_{z x}^{H}$ holds at any vector $(z, x)$, where $M R S_{z x}^{i}$ is defined as $M R S_{z x}^{i} \equiv-\left(\partial v_{i} / \partial z_{i}\right) /\left(\partial v_{i} / \partial x_{i}\right)$. This implies that for any income tax function the high-able individual does not choose to earn less income than the low-able.

The government is restricted by a budget constraint which reads

$$
\pi_{L}\left(z_{L}-x_{L}\right)+\pi_{H}\left(z_{H}-x_{H}\right)+\sum_{j=1,2,3} \tau_{j}\left(\pi_{L} c_{j L}+\pi_{H} c_{j H}\right) \geq g
$$

i.e. tax revenues have to be raised to finance exogenous public spending $g$. In addition the government is constrained by a self-selection constraint given by

$$
v_{H}\left(x_{H}, z_{H}, p_{1}, p_{2}, p_{3}, \overline{c_{1 H}}, \overline{c_{2 H}}\right) \geq v_{H}\left(x_{L}, z_{L}, p_{1}, p_{2}, p_{3}, \overline{c_{1 L}}, \overline{c_{2 L}}\right) \text {. }
$$

It guarantees that the high-able individual does not prefer the bundle which is designed for the low-able individual. The constraint that the $L$-type does not mimic the $H$ type is not binding in the optimum and therefore neglected, given that we restrict the analysis to cases, where the government wants to redistribute from high- to lowability persons. Observe from (8) that we assume that the reference levels for the mimicker and the L-types are identical, i.e. type $H$ when mimicking compares his/her consumption to $\overline{c_{k L}}, k=1,2$. Hence, we assume that income (and not ability) is 
decisive for the chosen reference level. ${ }^{11}$ To abbreviate notation indirect utility of the mimicker is denoted by $v_{H}[L]$ and consumption of the mimicker by $c_{j H}[L]$.

In addition the definitions of the reference levels given by (1) and (2) are taken into account as separate (equality) constraints. The Lagrange multipliers for the budget and the self-selection constraint are denoted by $\lambda$ and $\mu$, respectively, and the multipliers for the reference levels are given by $\gamma_{1 L}, \gamma_{1 H}, \gamma_{2 L}, \gamma_{2 H}$. The Lagrangian function and the first-order conditions for this maximization problem are provided in the technical Appendix.

\section{Optimal commodity tax structure}

As mentioned in the introduction, the additivity property is generally violated if individuals of different ability are not equally effective as externality-generating units. Then a role for other tax instruments arises to internalize the externality (Micheletto 2008). We generalize this discussion to a setting where there are two externalitygenerating goods, while the previous literature considered only one externality generating good. This allows us to analyze the interdependence of the two externalitygenerating commodities and its consequences for the optimal tax structure. Further, we focus on positional externalities, where it in fact matters which individual increases with his/her consumption the level of the externality (see equations 1 and 2).

We show in the technical Appendix that optimal commodity tax rates have to satisfy

$$
\begin{aligned}
& A\left(\begin{array}{c}
\tau_{1} \\
\tau_{2} \\
\tau_{3}
\end{array}\right)= \\
& \left(\begin{array}{l}
-\frac{\mu}{\lambda} \frac{\partial v_{H}[L]}{\partial x_{L}}\left(c_{1 H}[L]-c_{1 L}\right)+\sum_{k} \sum_{i}\left(\frac{\gamma_{k L}}{\lambda} \alpha_{k i}(L) \pi_{i} \frac{\partial c_{k i}^{c o m}}{\partial p_{1}}+\frac{\gamma_{k H}}{\lambda} \alpha_{k i}(H) \pi_{i} \frac{\partial c_{k i}^{c o m}}{\partial p_{1}}\right) \\
-\frac{\mu}{\lambda} \frac{\partial v_{H}[L]}{\partial x_{L}}\left(c_{2 H}[L]-c_{2 L}\right)+\sum_{k} \sum_{i}\left(\frac{\gamma_{k L}}{\lambda} \alpha_{k i}(L) \pi_{i} \frac{\partial c_{k i}^{\circ o m}}{\partial p_{2}}+\frac{\gamma_{k H}}{\lambda} \alpha_{k i}(H) \pi_{i} \frac{\partial c_{k i}}{\partial p_{2}}\right) \\
-\frac{\mu}{\lambda} \frac{\partial v_{H}[L]}{\partial x_{L}}\left(c_{3 H}[L]-c_{3 L}\right)+\sum_{k} \sum_{i}\left(\frac{\gamma_{k L}}{\lambda} \alpha_{k i}(L) \pi_{i} \frac{\partial c_{k i}^{c o m}}{\partial p_{3}}+\frac{\gamma_{k H}}{\lambda} \alpha_{k i}(H) \pi_{i} \frac{\partial c_{k i}^{\circ m}}{\partial p_{3}}\right)
\end{array}\right),
\end{aligned}
$$

where compensated demand for commodity $j$ of an individual $i$ is denoted by $c_{j i}^{\text {com }}$ and where

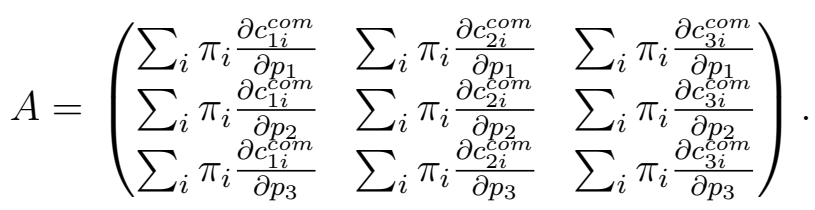

From equation (9) it can be seen that the shadow prices of the externalities (measured in terms of the government's tax revenues) $\gamma_{k i} / \lambda, k=1,2$ and $i=L, H$, play a central role in the determination of optimal commodity tax rates. They can be interpreted as the social harm $\left(\gamma_{k i} / \lambda>0\right)$ or gain $\left(\gamma_{k i} / \lambda<0\right)$ of a marginal increase in $\overline{c_{k i}}$.

${ }^{11}$ A similar assumption is made by Micheletto (2010). However, one could also think of a situation where the mimicker continues to compare his/her consumption to the reference levels of the $H$ type. As our main results remain valid under both variants, we chose the one that seems more plausible to us. 
Contrary to intuition, these shadow prices need not necessarily be positive. We show in the Appendix that due to a potential effect of a change in the reference level on the self-selection constraint and on the government's budget constraint, an increase in the reference level might actually lead to a social gain. However, the sign of these effects is ambiguous and due to a direct negative impact of an increase in the reference levels on the utility of individuals, a positive shadow price appears more plausible.

An implication of our assumption that there are two different positional commodities is that in general the shadow prices of the two commodities depend on each other. That is, the social harm or gain of a specific externality also depends on the social harm or gain of the other externalities, at least if compensated demand for the positional goods react to a change in the reference level of the respective other positional good. ${ }^{12}$ Lemma 1 summarizes the results on $\gamma_{k i} / \lambda$.

Lemma 1: The shadow prices of the externalities measured in terms of the government's revenues can be either positive or negative, although status consumption causes a negative externality. Further, if $\partial c_{m i}^{c o m} / \partial \overline{c_{k i}} \neq 0$ the shadow prices of the two positional commodities depend on each other, i.e. $\gamma_{k i} / \lambda$ depends on $\gamma_{m i} / \lambda$, with $k \neq m$ and $k, m=1,2$.

Proof: See the Appendix.

By applying Cramer's rule to the system of equations given in (9) one can derive an implicit solution for $\tau_{1}, \tau_{2}, \tau_{3}$, which is provided in the Appendix (see equations (21) and (24)). There we show that in general optimal commodity taxes, including the tax on the nonpositional good, depend in a complex way on all shadow prices $\gamma_{1 i} / \lambda$ and $\gamma_{2 i} / \lambda, i=L, H$. Thus, the additivity property does not hold in our model. It is optimal for the government to use all available commodity taxes to internalize the positional externalities. To gain intuition for this result, we provide conditions which are sufficient to restore the additivity property, as then it can be seen which aspects of the model drive this result. First, consider the optimal tax rate on the nonpositional good.

Proposition 1: In general the optimal tax on the nonpositional commodity $\tau_{3}^{*}$ serves to correct for the externalities induced by the consumption of commodities 1 and 2 which violates the additivity property. $\tau_{3}^{*}$ is not used to correct for the positional externalities if at least one of the following properties holds:

(i) equal weights $\alpha_{k L}(i)=\alpha_{k H}(i), k=1,2$ and $i=L, H$,

(ii) no compensated cross-price effects,

(iii) $\frac{\partial c_{L}^{c o m}}{\partial p_{j}} / \frac{\partial c_{k L}^{c o m}}{\partial p_{m}}=\frac{\partial c_{k H}^{c o m}}{\partial p_{j}} / \frac{\partial c_{k H}^{c o m}}{\partial p_{m}}, k=1,2$ and $j, m=1,2,3$.

Proof: See the Appendix.

Each condition (i)-(iii) is sufficient to restore the additivity property with respect to the tax rate on the nonpositional good. ${ }^{13}$ Then $\tau_{3}^{*}$ depends only on the well-known

\footnotetext{
${ }^{12}$ For the case of atmospheric externalities this result has originally been shown by Eckerstorfer (2012).

${ }^{13}$ Note that if all properties (i)-(iii) do not hold, one gets only a necessary condition for $\tau_{3}$ to contain
} 
self-selection part of commodity taxation which we denote by $\psi_{j}, j=1,2,3$ (a formal definition of $\psi_{3}$ is provided in the Appendix in equation (22)). That is, if at least one of (i)-(iii) holds

$$
\tau_{3}^{*}=\psi_{3} .
$$

$\psi_{3}$, and thus $\tau_{3}^{*}$, is zero if preferences are weakly separable in labor supply and consumption (Atkinson and Stiglitz 1976).

Next, consider the optimal tax rates on the positional commodities. As stated above, in general also the tax rates on the positional commodities depend in a complex way on all four shadow prices, in addition to the self-selection part of commodity taxation (see equation (24) in the Appendix). Remarkably, the shadow prices of the respective other externality-generating commodity appear explicitly in the optimal tax formula for a positional good. This is also in contradiction to the additivity property which states that the presence of an externality only alters the tax formula on that particular good. But again each of the conditions (i)-(iii) provided in Proposition 1 is sufficient for the part in the optimal tax formula depending on the shadow price of the other positional good to be zero. That is, then the optimal $\tau_{k}^{*}$ depends only on a correction term containing $\gamma_{k i} / \lambda$ and on the self-selection term $\psi_{k}$. Note, however, from Lemma 1 we know that even then $\gamma_{m i} / \lambda$ has an indirect impact on $\tau_{k}$ through its effect on $\gamma_{k} / \lambda$. This result is summarized in Proposition 2.

Proposition 2: In general the optimal tax rates on the positional commodities $\tau_{k}^{*}$, $k=1,2$, depend explicitly on the shadow prices of both externalities. This violates the additivity property. $\tau_{k}^{*}$ is not affected directly by $\gamma_{m i} / \lambda$, with $k \neq m$ and $k, m=1,2$, if at least one of the properties (i)-(iii) from Proposition 1 holds. Then $\gamma_{m i} / \lambda$ affects $\tau_{k}^{*}$ only indirectly through its effect on $\gamma_{k} / \lambda$ (see Lemma 1 ).

Proof: See the Appendix.

From Propositions 1 and 2 we can conclude that in general the full set of commodity taxes should be used to internalize the externalities. The intuition for this result is as follows. If the consumption of the two types is weighted differently in the formation of the reference levels (property (i) does not hold, i.e. $\alpha_{k L}(i) \neq \alpha_{k H}(i)$ ) the government would want to tax the status consumption of the two types at different rates, which is, however, not possible as $\tau_{1}$ and $\tau_{2}$ are restricted to be proportional. As a consequence the government wants to exploit other possibilities to channel the consumption decision of individuals in the desired direction. This is possible with the full set of commodity taxes if compensated cross-price effects are present (property (ii) does not hold) and if the two types react differently to relative price changes (property (iii) does not hold). For example, if introducing a tax on the nonpositional commodity modifies the consumption baskets of the two types such that the type, whose consumption is weighted stronger in the formation of the reference levels, consumes relatively more

Pigouvian elements. That is, even if all of (i)-(iii) do not hold it cannot be excluded that the Pigouvian part in the optimal tax formula cancels out as there are both positive and negative terms. 
of the nonpositional good than the other type after the introduction of the tax, then $\tau_{3}$ serves to correct for the externalities. Concerning the sign of optimal commodity tax rates not much can be said in particular with respect to $\tau_{3}$. Also the tax rates on the positional commodities can in principle have either sign, but if shadow prices are positive $\tau_{1}$ and $\tau_{2}$ are likely to be positive.

If one assumes that the consumption of the two types is weighted equally in the formation of the reference levels, i.e. $\alpha_{k L}(i)=\alpha_{k H}(i)=\alpha_{k}(i)$ (property (i) holds), $k=1,2$ and $i=L, H$, then $\tau_{k}^{*}$ reduces to

$$
\tau_{k}^{*}=\psi_{k}+\frac{\gamma_{k L}}{\lambda} \alpha_{k}(L)+\frac{\gamma_{k H}}{\lambda} \alpha_{k}(H),
$$

$k=1,2$. In this case the optimal tax formula looks similar to those provided by Pirttilä and Tuomala (1997) and Cremer et al. (1998) where the externality is assumed to be of the atmospheric type. The main difference is that even with equal weights the highand the low-able still might face different externalities in our framework, and hence the Pigouvian part in (12) consists of a weighted sum of the shadow prices for the two income groups.

Note that two possible variants of our model that are frequently discussed in the literature are upward and within-group comparison. Both of them can easily be considered in our model just by adapting weights properly. In particular upward comparison received a lot of attention from both the theoretical and empirical literature. ${ }^{14}$ In this case the reference levels are given by $\overline{c_{k L}}=\alpha_{k H}(L) \pi_{H} c_{k H}$ and $\overline{c_{k H}}=\alpha_{k H}(H) \pi_{H} c_{k H}$, i.e. only the consumption of the $H$-types is relevant in the formation of the reference levels. ${ }^{15}$ In the case of within-group comparison individuals compare their consumption with the consumption of individuals in the same income group. Then the reference levels are given by $\overline{c_{k H}}=\alpha_{k H}(H) \pi_{H} c_{k H}$ and $\overline{c_{k L}}=\alpha_{k L}(L) \pi_{L} c_{k L}$. Observe that in case of upward as well as within-group comparison the consumption of the two types is weighted differently in the formation of the reference levels. Hence, it follows from Proposition 1 and 2 that for these special cases it is in general optimal to use all available commodity taxes to internalize the externalities induced by the consumption of commodities 1 and 2. Clearly, for this statement to be true it is essential that properties (ii)-(iii) do not hold.

\section{Optimal income tax structure}

In the previous section we have established the result that it is in general optimal to use the whole set of commodity taxes, notably also the tax on the nonpositional good, in order to correct for the externalities. In this section we analyze the potential role of the income tax to serve for the same purpose, that is, we want to find out how the

\footnotetext{
${ }^{14}$ See for example Micheletto (2010) and Aronsson and Johansson-Stenman (2010) for theoretical studies assuming upward comparison and Bowles and Park (2005) for an empirical study supporting this assumption.

${ }^{15}$ An alternaitve approach to model upward comparison would be to assume that only the $L$-types compare their consumption to the one of the $H$ types while the $H$-types themselves have no positional concerns. In that case $\alpha_{k H}(H)=0$.
} 
presence of the externalities affects the optimal income tax schedule. First, we analyze the optimal income tax in the presence of differential commodity taxation and then for the case when commodity taxes are restricted to be uniform.

\subsection{Marginal income tax rates in the presence of differential commodity taxes}

The marginal income tax rates can easily be obtained by dividing the first-order conditions for $z_{i}$ and $x_{i}((32)$ by (30) and (31) by (29)) and by using equation (5). The intuition is that the government chooses the optimal gross and net income for each individual and then determines an income tax function such that individuals realize those bundles. In the technical Appendix we show that the optimal marginal income tax rates for arbitrary commodity taxes are given by

$$
\begin{aligned}
T^{\prime}\left(z_{H}\right)= & \sum_{k=1,2}\left(\frac{\gamma_{k L}}{\lambda} \alpha_{k H}(L)+\frac{\gamma_{k H}}{\lambda} \alpha_{k H}(H)-\tau_{k}\right)\left(\frac{\partial c_{k H}}{\partial z_{H}}+M R S_{z x}^{H} \frac{\partial c_{k H}}{\partial x_{H}}\right) \\
& -\tau_{3}\left(\frac{\partial c_{3 H}}{\partial z_{H}}+M R S_{z x}^{H} \frac{\partial c_{3 H}}{\partial x_{H}}\right) \\
T^{\prime}\left(z_{L}\right)= & \sum_{k=1,2}\left(\frac{\gamma_{k L}}{\lambda} \alpha_{k L}(L)+\frac{\gamma_{k H}}{\lambda} \alpha_{k L}(H)-\tau_{k}\right)\left(\frac{\partial c_{k L}}{\partial z_{L}}+M R S_{z x}^{L} \frac{\partial c_{k L}}{\partial x_{L}}\right) \\
& -\tau_{3}\left(\frac{\partial c_{3 L}}{\partial z_{L}}+M R S_{z x}^{L} \frac{\partial c_{3 L}}{\partial x_{L}}\right)+\frac{\mu}{\lambda \pi_{L}} \frac{\partial v_{H}[L]}{\partial x_{L}}\left(M R S_{z x}^{L}-M R S_{z x}^{H}[L]\right) .
\end{aligned}
$$

From (13) and (14) it can be concluded that in general also the marginal income tax rates are affected by the externalities, which implies that the additivity property is also violated with respect to the income tax. This can immediately be seen by plugging in the optimal commodity tax rates given by (21) and (24) into (13) and (14). Then one observes that the optimal income tax schedule depends explicitly on all shadow prices of the externalities. If, however, $\alpha_{k H}(i)=\alpha_{k L}(i), k=1,2$ and $i=L, H$ (property (i) in Proposition 1), then the additivity property is restored with respect to the income tax as then the Pigouvian elements (the sum term) in (13) and (14) cancel out. Note that with equal weights optimal commodity tax rates on goods 1 and 2 are given by (12) and the tax rate on the nonpositional good by (11), from which it follows that the Pigouvian parts in (13) and (14) drop out.

An interesting difference between the results concerning the income tax and the tax on the nonpositional good is that from Proposition 1 we know that even if the two types are not equally effective as externality-generating units, $\tau_{3}$ is unaffected by the externalities if there are no compensated cross price effects (property (ii)) and/or the proportions of compensated price effects (own to cross and cross to cross price effects) for commodities 1 and 2 are the same for both types (property (iii)). However, the same is not true for the marginal income tax rates which are influenced by the externalities as soon as $\alpha_{k H}(i) \neq \alpha_{k L}(i)$. A closely related result is due to Micheletto (2008) who showed that whenever the additivity property is violated with respect to the com- 
modity tax structure, also the income tax schedule contains Pigouvian elements, but that the reverse is not necessarily true. We have derived explicit conditions for this result. Proposition 3 summarizes our characterization of the income tax schedule.

Proposition 3: In general the optimal marginal income tax rates for both types depend on the externalities. Thus, the additivity property is also violated with respect to the income tax. A sufficient condition for the positional externalities to have no impact on the income tax is $\alpha_{k H}(i)=\alpha_{k L}(i), k=1,2$ and $i=L, H$.

Note that without further assumptions the sign of the Pigouvian parts in the income tax schedule is ambiguous, even if shadow prices are assumed to be positive. It depends on whether the optimal commodity tax rates $\tau_{k}, k=1,2$ are larger or smaller than the weighted sum of the shadow prices of the taxed commodity $k$, on whether the status goods are complements or substitutes to leisure $\left(\partial c_{k i} / \partial z_{i} \gtrless 0\right)$ and on whether demand for the status goods increases with net income $\left(\partial c_{k i} / \partial x_{i} \gtrless 0\right)$. In the next subsection we discuss the optimal income tax schedule if commodity taxes are restricted to be uniform. For this scenario more precise statements concerning the sign and the shape of the Pigouvian parts in the marginal income tax rates can be made.

\subsection{Marginal income tax rates with uniform taxation of commodities}

One potential objection against our results is that differential taxation of status goods might not be feasible for political economy reasons. ${ }^{16}$ In this subsection we touch upon this issue and assume that total consumption is restricted to be taxed uniformly at a rate $\tau$, i.e. differential taxation of status goods is no longer possible. Without loss of generality we set $\tau=0$ since the effect of any uniform consumption tax can also be attained through the income tax. Given this restriction on the commodity tax structure we analyze the role of the income tax to internalize the positional externalities. The optimal marginal income tax rates can again be obtained by combining the first-order-conditions for $x_{i}, z_{i}$ with equation (5) and by taking into account the restriction that $\tau=0$. From (13) and (14) it follows that in this case the optimal marginal income tax rates are given by

$$
\begin{aligned}
T^{\prime}\left(z_{H}\right)= & \sum_{k=1,2}\left(\frac{\gamma_{k L}}{\lambda} \alpha_{k H}(L)+\frac{\gamma_{k H}}{\lambda} \alpha_{k H}(H)\right)\left(\frac{\partial c_{k H}}{\partial z_{H}}+M R S_{z x}^{H} \frac{\partial c_{k H}}{\partial x_{H}}\right), \\
T^{\prime}\left(z_{L}\right)= & \sum_{k=1,2}\left(\frac{\gamma_{k L}}{\lambda} \alpha_{k L}(L)+\frac{\gamma_{k H}}{\lambda} \alpha_{k L}(H)\right)\left(\frac{\partial c_{k L}}{\partial z_{L}}+M R S_{z x}^{L} \frac{\partial c_{k L}}{\partial x_{L}}\right) \\
& +\frac{\mu}{\lambda \pi_{L}} \frac{\partial v_{H}[L]}{\partial x_{L}}\left(M R S_{z x}^{L}-M R S_{z x}^{H}[L]\right) .
\end{aligned}
$$

\footnotetext{
${ }^{16}$ This point has been raised for example by Ireland (2001) and Frank $(1999,2008)$.
} 
In the abscence of commodity taxes only the income tax can be used to correct for the positional externalities. Hence, the income tax is affected by the externalities no matter how the reference level looks like. But surprisingly, the sign of the corrective parts in (15) and (16) (sum term) is still ambiguous, even if shadow prices are positive. This ambiguity in the sign of the Pigouvian part in the income tax schedule is an important difference to the results in previous studies on the optimal income tax when relative consumption matters, which assume that there is only one consumption good (see for example Aronsson and Johansson-Stenman 2008). There, as soon as the shadow price is positive, the Pigouvian part in the income tax schedule is also positive. ${ }^{17}$ Things become more complicated if there are both positional and nonpositional forms of consumption. For instance, if a status good is a complement to leisure, and hence $\partial c_{k i} / \partial z_{i}<0$, then there is an effect that works in the opposite direction requiring a lower or even negative marginal income tax rate. As an example think of playing golf or visiting an expensive restaurant, which are complementary to leisure and have some status character. The intuition for this effect is that increasing the marginal income tax rate induces individuals to enjoy more leisure and, thus, to also consume more of the status good (e.g. play more golf). Clearly, if the status good is a normal good, and hence $\partial c_{k i} / \partial x_{i}>0$, this effect is partly offset by the effect of a reduction in net income on the demand for the status good. The point is that if individuals can spend their income on positional and nonpositional goods, a higher income tax does not necessarily imply lower consumption of the positional good, which is in contrast to a model where all consumption is assumed to be positional. This result is stated in Proposition 4.

Proposition 4: Assume that shadow prices are positive and that commodities 1 and 2 are normal goods, i.e. $\gamma_{k i} / \lambda>0$ and $\partial c_{k i} / \partial x_{i}>0$ with $k=1,2$ and $i=L, H$. Then the Pigouvian parts in the income tax schedule are unambiguously positive if demand for commodities 1 and 2 is either unaffected by leisure or decreases with leisure $\left(\partial c_{k i} / \partial z_{i} \geq 0\right)$. If, however, the demand for status goods increases with leisure $\left(\partial c_{k i} / \partial z_{i}<0\right)$ the sign of the Pigouvian part is ambiguous.

Finally, we show that with some additional assumptions the Pigouvian part in the income tax schedule is progressive. The Pigouvian elements in (15) and (16) are given by the first term on the RHS of these equations. Assuming that the consumption of the $H$-type has a larger weight in the formation of the reference levels (e.g. upward comparison), i.e. $\alpha_{k H}(i)>\alpha_{k L}(i), i=1,2$, the inequality

$$
\sum_{k=1,2}\left(\frac{\gamma_{k L}}{\lambda} \alpha_{k H}(L)+\frac{\gamma_{k H}}{\lambda} \alpha_{k H}(H)\right)>\sum_{k=1,2}\left(\frac{\gamma_{k L}}{\lambda} \alpha_{k L}(L)+\frac{\gamma_{k H}}{\lambda} \alpha_{k L}(H)\right)
$$

\footnotetext{
${ }^{17}$ Their formulas for the marginal income tax rates do not include a shadow price. In their notation, a sufficient condition for the relative consumption concerns to contribute to higher marginal income tax rates is that the low-ability type is at least as positional as the mimicker. In the absence of commodity taxes this condition would imply positive shadow prices in our framework, which justifies this statement.
} 
holds, provided that the shadow prices are positive. Given $\alpha_{k H}(i)>\alpha_{k L}(i)$ a sufficient condition for the Pigouvian element in the optimal marginal income tax rate to be larger for the $H$-type (see (15) and (16)), and hence, for the Pigouvian parts in the income tax schedule to be progressive, is

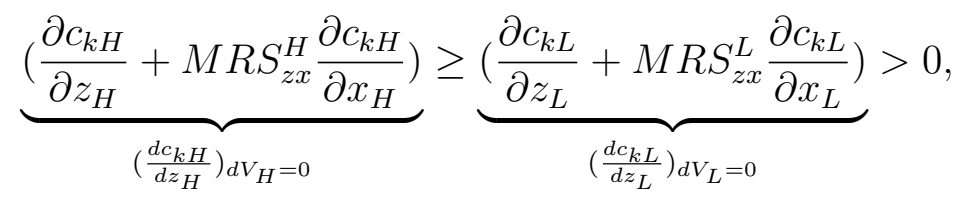

$k=1,2$. This condition states that the $H$-types change their demand for the positional commodities at least as much as the $L$-types, in case of a marginal increase of gross income which is compensated such that their utility does not change. Further, this change in demand has to be positive, which is guaranteed if commodity $k$ is a normal good and if demand for $k$ is unaffected by leisure or a substitute to leisure. Observe that if preferences are weakly separable in labor supply and consumption and homothetic in consumption this condition reduces to $M R S_{z x}^{H} \geq M R S_{z x}^{L}$ at the optimal bundles $\left(z_{H}, x_{H}\right),\left(z_{L}, x_{L}\right)$ as with such preferences $\partial c_{k i} / \partial z_{i}=0$ and $\partial c_{k H} / \partial x_{H}=\partial c_{k L} / \partial x_{L}$. In the absence of externalities the inequality $M R S_{z x}^{H} \geq M R S_{z x}^{L}$ holds at the optimal allocation, which is an immediate consequence of the zero at the top result in the standard version of the Mirrlees income tax model (Sadka 1976, Seade 1977). But in our model this inequality can in principle be violated as it is optimal to also distort the decision of the $H$ types. Nevertheless, if the social damage caused by the externality is small, this condition is likely to hold. The result concerning the progressivity of the Pigouvian part in the income tax schedule is summarized in Proposition 5.

Proposition 5: Assume that $\alpha_{k H}(i)>\alpha_{k L}(i), k=1,2$ and $i=L, H$. Then, if the externality is socially harmful (positive shadow prices), equation (18) is a sufficient but not necessary condition for the Pigouvian parts in the optimal income tax schedule to be progressive.

Thus, if the consumption of the $H$-type has a larger weight in the formation of the externality, then it is optimal for the Pigouvian element in the income tax schedule to be larger for the $H$-type, at least if the consumption of commodities 1 and 2 of the high-able responds at least as strong to a change in gross-income than that of the low-able.

\section{Conclusion}

In this paper we have studied the optimal taxation of income and commodities when individuals make relative consumption comparisons. In contrast to previous studies we have assumed that there are both positional and nonpositional forms of consumption, taking into account the idea that relative concerns matter for some but not for all commodities. Further, we have modeled the reference consumption levels in a very general way. We take into account that different individuals might 
have different reference levels and that the consumption of some individuals might be more important in the formation of the reference levels than the consumption of other individuals. These extensions have some interesting implications for the optimal tax structure.

We have found that in general the whole tax system is affected by the positional externalities. In particular even the tax rate on the nonpositional good and the income tax serve for externality-correcting purposes at least if the consumption of the two types is weighted differently in the formation of the reference levels (e.g. in case of upward comparison). The reason is that in this case the proportional taxes on the positional commodities alone cannot achieve the additional differentiation between the two types which then becomes desirable. Some additional differentiation can be attained with the whole set of commodity taxes provided that cross-price effects are present and that the two types react differently to relative price changes. Further differentiation can be achieved with the nonlinear income tax as it allows to tax the two types differently.

If commodity taxes are restricted to be uniform for example due to political economy reasons, then only the income tax can serve to correct for the positional externalities. In general the optimal marginal income tax rates are higher than in the absence of relative concerns and the well-known zero at the top result does not hold. However, our assumption that individuals can spend their income on both positional and nonpositional forms of consumption implies that if status consumption is a complement to leisure then the sign of the Pigouvian part in the income tax is ambiguous. The reason is that in this case an increase in the marginal income tax rate does not necessarily lead to a decline in the demand for status goods. Moreover we have shown that if the consumption of the high-able individuals has a higher weight in the formation of the reference levels then with some mild additional assumptions the Pigouvian elements in the marginal income tax rates are progressive.

\section{Appendix}

\section{Proof of Lemma 1}

We show in the technical Appendix that the shadow prices measured in terms of tax revenues can be expressed as

$$
\begin{aligned}
\frac{\gamma_{k L}}{\lambda}= & \frac{1}{1-\alpha_{k L}(L) \pi_{L} \frac{\partial c_{k L}^{c o m}}{\partial \overline{c_{k L}}}}\left(\pi_{L} M W P_{k L}-\mu \frac{\partial v_{H}[L]}{\partial x_{L}}\left(M W P_{k H}[L]-M W P_{k L}\right)\right. \\
& -\pi_{L} \sum_{j=1}^{3} \tau_{j} \frac{\partial c_{j L}^{c o m}}{\partial \overline{c_{k L}}}+\frac{\gamma_{m L}}{\lambda} \alpha_{m L}(L) \pi_{L} \frac{\partial c_{m L}^{c o m}}{\partial \overline{c_{k L}}}+\sum_{m=1,2} \frac{\gamma_{m H}}{\lambda} \alpha_{m L}(H) \pi_{L} \frac{\partial c_{m L}^{c o m}}{\partial \overline{c_{k L}}} \gamma(19)
\end{aligned}
$$

$k, m=1,2$ and $k \neq m$, and where $M W P_{k i} \equiv-\frac{\partial v_{i} / \partial \overline{c_{k i}}}{\partial v_{i} / \partial x_{i}}$ is the marginal willingness to pay of an individual $i$ to reduce the reference consumption level $\overline{c_{k i}}$ by one unit. The first factor on the RHS of (19) is the so called feedback effect which captures the effect of the level of the externality on compensated demand of the commodity which 
generates that externality (see also Bovenberg and Goulder (2002) for a discussion). It is assumed to be positive in order for the model to be stable. The first term within parenthesis accounts for the direct negative impact of the externality which increases the shadow price. The second and the third term within parenthesis capture the impact of the reference level on the self-selection and the budget constraint, respectively. The sign of both effects is ambiguous. Finally, the two terms in the second line of (19) show that the various shadow prices of the externalities depend on each other (again with ambiguous signs). All together, $\gamma_{k L}$ can be either positive or negative.

Similarly, we show in the technical Appendix that $\gamma_{k H} / \lambda$ can be expressed as

$$
\begin{aligned}
\frac{\gamma_{k H}}{\lambda}= & \frac{1}{1-\alpha_{k H}(H) \pi_{H} \frac{\partial c_{k H}^{c o m}}{\partial \bar{c}_{k H}}}\left(\pi_{H} M W P_{k H}-\pi_{H} \sum_{j=1}^{3} \tau_{j} \frac{\partial c_{j H}^{c o m}}{\partial \overline{c_{k H}}}+\frac{\gamma_{m H}}{\lambda} \alpha_{m H}(H) \pi_{H} \frac{\partial c_{m H}^{c o m}}{\partial \overline{c_{k H}}}\right. \\
& \left.+\sum_{m=1,2} \frac{\gamma_{m L}}{\lambda} \alpha_{m H}(L) \pi_{H} \frac{\partial c_{m H}^{c o m}}{\partial \overline{c_{k H}}}\right),
\end{aligned}
$$

$k, m=1,2$ and $k \neq m$. The terms in (20) have a similar interpretation as the terms in (19). Thus, $\gamma_{k H} / \lambda$ can be either positive or negative and is also affected by the other shadow prices. Note, that (20) contains no effect on the self-selection constraint. This is because of our assumption that a high-able individual when mimicking compares his/her consumption to the reference levels of the $L$ types.

\section{Proof of Proposition 1}

By applying Cramer's rule to the system of equations given in equation (9) one can derive implicit solutions for the optimal commodity tax rates. The optimal tax on the nonpositional good is implicitly given by

$$
\tau_{3}^{*}=\psi_{3}+\frac{\pi_{L} \pi_{H}}{|A|} \sum_{k=1,2}\left[\frac{\gamma_{k L}}{\lambda}\left(\alpha_{k H}(L)-\alpha_{k L}(L)\right)+\frac{\gamma_{k H}}{\lambda}\left(\alpha_{k H}(H)-\alpha_{1 L}(H)\right)\right] D_{k},(21)
$$

where $|A|$ is the determinant of matrix $A$ (equation (10)) and where $\psi_{3}$ and $D_{k}$ are defined as

$$
\begin{aligned}
\psi_{3} \equiv & \frac{1}{|A|}\left[\frac { \mu } { \lambda } \frac { \partial v _ { H } [ L ] } { \partial x _ { L } } \left(-\left(c_{3 H}[L]-c_{3 L}\right)\left(\sum_{i} \pi_{i} \frac{\partial c_{1 i}^{c o m}}{\partial p_{1}} \sum_{i} \pi_{i} \frac{\partial c_{2 i}^{c o m}}{\partial p_{2}}\right.\right.\right. \\
& \left.-\sum_{i} \pi_{i} \frac{\partial c_{1 i}^{c o m}}{\partial p_{2}} \sum_{i} \pi_{i} \frac{\partial c_{2 i}^{c o m}}{\partial p_{1}}\right)+\left(c_{1 H}[L]-c_{1 L}\right)\left(\sum_{i} \pi_{i} \frac{\partial c_{2 i}^{c o m}}{\partial p_{2}} \sum_{i} \pi_{i} \frac{\partial c_{1 i}^{c o m}}{\partial p_{3}}\right. \\
& \left.-\sum_{i} \pi_{i} \frac{\partial c_{1 i}^{c o m}}{\partial p_{2}} \sum_{i} \pi_{i} \frac{\partial c_{2 i}^{c o m}}{\partial p_{3}}\right)+\left(c_{2 H}[L]-c_{2 L}\right)\left(\sum_{i} \pi_{i} \frac{\partial c_{1 i}^{c o m}}{\partial p_{1}} \sum_{i} \pi_{i} \frac{\partial c_{2 i}^{c o m}}{\partial p_{3}}\right. \\
& \left.\left.\left.-\sum_{i} \pi_{i} \frac{\partial c_{2 i}^{c o m}}{\partial p_{1}} \sum_{i} \pi_{i} \frac{\partial c_{1 i}^{c o m}}{\partial p_{3}}\right)\right)\right],
\end{aligned}
$$




$$
\begin{aligned}
D_{k} \equiv & \sum_{i} \pi_{i} \frac{\partial c_{n i}^{c o m}}{\partial p_{n}}\left(\frac{\partial c_{k L}^{c o m}}{\partial p_{k}} \frac{\partial c_{k H}^{c o m}}{\partial p_{3}}-\frac{\partial c_{k H}^{c o m}}{\partial p_{k}} \frac{\partial c_{k L}^{c o m}}{\partial p_{3}}\right)+\sum_{i} \pi_{i} \frac{\partial c_{n i}^{c o m}}{\partial p_{k}}\left(\frac{\partial c_{k L}^{c o m}}{\partial p_{3}} \frac{\partial c_{k H}^{c o m}}{\partial p_{n}}\right. \\
& \left.-\frac{\partial c_{k H}^{c o m}}{\partial p_{3}} \frac{\partial c_{k L}^{c o m}}{\partial p_{n}}\right)+\sum_{i} \pi_{i} \frac{\partial c_{n i}^{c o m}}{\partial p_{3}}\left(\frac{\partial c_{k L}^{c o m}}{\partial p_{n}} \frac{\partial c_{k H}^{c o m}}{\partial p_{k}}-\frac{\partial c_{k H}^{c o m}}{\partial p_{n}} \frac{\partial c_{k L}^{c o m}}{\partial p_{k}}\right)
\end{aligned}
$$

with $k, n=1,2$ and $k \neq n$.

From (21) it can be seen that in general $\tau_{3}^{*}$ depends on $\gamma_{k i}, k=1,2$ and $i=L, H$, violating the additivity property. If the consumption of both types is weighted equally in the formation of the reference levels (property (i)), one can see that the Pigouvian part in (21) (second term) vanishes. This is also the case if there are no compensated cross price effects (property (ii)) and/or the proportions of compensated price effects (own to cross and cross to cross price effects) for commodities 1 and 2 are the same for both types (property (iii)), as then $D_{k}$ as given by (23) is zero.

\section{Proof of Proposition 2}

The optimal tax rates on the positional commodities are implicitly given by

$$
\begin{aligned}
\tau_{k}^{*}= & \psi_{k}+\frac{1}{|A|}\left(\frac{\gamma_{k L}}{\lambda} E_{k L}+\frac{\gamma_{k H}}{\lambda} E_{k H}\right) \\
& +\frac{\pi_{L} \pi_{H}}{|A|}\left[\frac{\gamma_{m L}}{\lambda}\left(\alpha_{m H}(L)-\alpha_{m L}(L)\right)+\frac{\gamma_{m H}}{\lambda}\left(\alpha_{m H}(H)-\alpha_{m L}(H)\right)\right] F_{k},
\end{aligned}
$$

with $k, m=1,2$ and $k \neq m$, and where $\psi_{k}, E_{k s}, s=L, H$, and $F_{k}$ are defined by

$$
\begin{aligned}
\psi_{k} \equiv & \frac{1}{|A|}\left[\frac { \mu } { \lambda } \frac { \partial v _ { H } [ L ] } { \partial x _ { L } } \left(-\left(c_{k H}[L]-c_{k L}\right)\left(\sum_{i} \pi_{i} \frac{\partial c_{m i}^{c o m}}{\partial p_{m}} \sum_{i} \pi_{i} \frac{\partial c_{3 i}^{c o m}}{\partial p_{3}}\right.\right.\right. \\
& \left.-\sum_{i} \pi_{i} \frac{\partial c_{m i}^{c o m}}{\partial p_{3}} \sum_{i} \pi_{i} \frac{\partial c_{3 i}^{c o m}}{\partial p_{m}}\right)+\left(c_{m H}[L]-c_{m L}\right)\left(\sum_{i} \pi_{i} \frac{\partial c_{m i}^{c o m}}{\partial p_{k}} \sum_{i} \pi_{i} \frac{\partial c_{3 i}^{c o m}}{\partial p_{3}}\right. \\
& \left.-\sum_{i} \pi_{i} \frac{\partial c_{3 i}^{c o m}}{\partial p_{k}} \sum_{i} \pi_{i} \frac{\partial c_{m i}^{c o m}}{\partial p_{3}}\right)+\left(c_{3 H}[L]-c_{3 L}\right)\left(\sum_{i} \pi_{i} \frac{\partial c_{m i}^{c o m}}{\partial p_{m}} \sum_{i} \pi_{i} \frac{\partial c_{3 i}^{c o m}}{\partial p_{k}}\right. \\
& \left.\left.\left.-\sum_{i} \pi_{i} \frac{\partial c_{m i}^{c o m}}{\partial p_{k}} \sum_{i} \pi_{i} \frac{\partial c_{3 i}^{c o m}}{\partial p_{m}}\right)\right)\right], \\
E_{k s} \equiv & \sum_{i} \alpha_{k i}(s) \pi_{i} \frac{\partial c_{k i}^{c o m}}{\partial p_{k}}\left(\sum_{i} \pi_{i} \frac{\partial c_{m i}^{c o m}}{\partial p_{m}} \sum_{i} \pi_{i} \frac{\partial c_{3 i}^{c o m}}{\partial p_{3}}-\sum_{i} \pi_{i} \frac{\partial c_{m i}^{c o m}}{\partial p_{3}} \sum_{i} \pi_{i} \frac{\partial c_{3 i}^{c o m}}{\partial p_{m}}\right) \\
& +\sum_{i} \alpha_{k i}(s) \pi_{i} \frac{\partial c_{k i}^{c o m}}{\partial p_{m}}\left(\sum_{i} \pi_{i} \frac{\partial c_{3 i}^{c o m}}{\partial p_{k}} \sum_{i} \pi_{i} \frac{\partial c_{m i}^{c o m}}{\partial p_{3}}-\sum_{i} \pi_{i} \frac{\partial c_{m i}^{c o m}}{\partial p_{k}} \sum_{i} \pi_{i} \frac{\partial c_{3 i}^{c o m}}{\partial p_{3}}\right) \\
& +\sum_{i} \alpha_{k i}(s) \pi_{i} \frac{\partial c_{k i}^{c o m}}{\partial p_{3}}\left(\sum_{i} \pi_{i} \frac{\partial c_{m i}^{c o m}}{\partial p_{k}} \sum_{i} \pi_{i} \frac{\partial c_{3 i}^{c o m}}{\partial p_{m}}\right. \\
& \left.-\sum_{i} \pi_{i} \frac{\partial c_{m i}^{c o m}}{\partial p_{m}} \sum_{i} \pi_{i} \frac{\partial c_{3 i}^{c o m}}{\partial p_{k}}\right),
\end{aligned}
$$




$$
\begin{aligned}
F_{k} \equiv & \sum_{i} \pi_{i} \frac{\partial c_{3 i}^{c o m}}{\partial p_{3}}\left(\frac{\partial c_{m L}^{c o m}}{\partial p_{m}} \frac{\partial c_{m H}^{c o m}}{\partial p_{k}}-\frac{\partial c_{m H}^{c o m}}{\partial p_{m}} \frac{\partial c_{m L}^{c o m}}{\partial p_{k}}\right)+\sum_{i} \pi_{i} \frac{\partial c_{3 i}^{c o m}}{\partial p_{m}}\left(\frac{\partial c_{m L}^{c o m}}{\partial p_{k}} \frac{\partial c_{m H}^{c o m}}{\partial p_{3}}\right. \\
& \left.-\frac{\partial c_{m H}^{c o m}}{\partial p_{k}} \frac{\partial c_{m L}^{c o m}}{\partial p_{3}}\right)+\sum_{i} \pi_{i} \frac{\partial c_{3 i}^{c o m}}{\partial p_{k}}\left(\frac{\partial c_{m L}^{c o m}}{\partial p_{3}} \frac{\partial c_{m H}^{c o m}}{\partial p_{m}}-\frac{\partial c_{m H}^{c o m}}{\partial p_{3}} \frac{\partial c_{m L}^{c o m}}{\partial p_{m}}\right) .
\end{aligned}
$$

From (24) it can be seen that in general $\tau_{k}^{*}, k=1,2$, depends explicitly on the shadow prices of both externalities, violating the additivity property. One can see immediately that if $\alpha_{m H}(i)=\alpha_{m L}(i)$ (implied by property (i)) the term depending explicitly on $\gamma_{k m} / \lambda$ is zero (second line in (24)). The same is true if there are no compensated cross price effects (property (ii)) and/or the proportions of compensated price effects (own to cross and cross to cross price effects) for commodities 1 and 2 are the same for both types (property (iii)), as then $F_{k}$ as given by (27) is zero.

\section{Technical Appendix}

\section{First-order conditions of the government's maximization problem}

The Lagrangian for the government's maximization problem reads

$$
\begin{aligned}
\mathcal{L}= & f_{L} v_{L}+f_{H} v_{H}+\lambda\left[\pi_{L}\left(z_{L}-x_{L}\right)+\pi_{H}\left(z_{H}-x_{H}\right)+\sum_{j=1,2,3} \tau_{j}\left(\pi_{L} c_{j L}+\pi_{H} c_{j H}\right)-g\right] \\
& +\mu\left(v_{H}-v_{H}[L]\right)+\gamma_{1 L}\left(\overline{c_{1 L}}-\alpha_{1 L}(L) \pi_{L} c_{1 L}-\alpha_{1 H}(L) \pi_{H} c_{1 H}\right)+\gamma_{1 H}\left(\overline{c_{1 H}}\right. \\
& \left.-\alpha_{1 L}(H) \pi_{L} c_{1 L}-\alpha_{1 H}(H) \pi_{H} c_{1 H}\right)+\gamma_{2 L}\left(\overline{c_{2 L}}-\alpha_{2 L}(L) \pi_{L} c_{2 L}-\alpha_{2 H}(L) \pi_{H} c_{2 H}\right) \\
& +\gamma_{2 H}\left(\overline{c_{2 H}}-\alpha_{2 L}(H) \pi_{L} c_{2 L}-\alpha_{2 H}(H) \pi_{H} c_{2 H}\right) .
\end{aligned}
$$

The first-order conditions with respect to the income bundles $x_{i}, z_{i}, i=L, H$, are given by:

$$
\begin{gathered}
f_{L} \frac{\partial v_{L}}{\partial x_{L}}=\lambda \pi_{L}-\lambda \pi_{L} \sum_{j=1,2,3} \tau_{j} \frac{\partial c_{j L}}{\partial x_{L}}+\mu \frac{\partial v_{H}[L]}{\partial x_{L}}+\pi_{L} \sum_{k=1,2} \sum_{i=L, H} \frac{\partial c_{k L}}{\partial x_{L}} \gamma_{k i} \alpha_{k L}(i), \\
f_{H} \frac{\partial v_{H}}{\partial x_{H}}=\lambda \pi_{H}-\lambda \pi_{H} \sum_{j=1,2,3} \tau_{j} \frac{\partial c_{j H}}{\partial x_{H}}-\mu \frac{\partial v_{H}}{\partial x_{H}}+\pi_{H} \sum_{k=1,2} \sum_{i=L, H} \frac{\partial c_{k H}}{\partial x_{H}} \gamma_{k i} \alpha_{k H}(i), \\
f_{L} \frac{\partial v_{L}}{\partial z_{L}}=-\lambda \pi_{L}-\lambda \pi_{L} \sum_{j=1,2,3} \tau_{j} \frac{\partial c_{j L}}{\partial z_{L}}+\mu \frac{\partial v_{H}[L]}{\partial z_{L}}+\pi_{L} \sum_{k=1,2} \sum_{i=L, H} \frac{\partial c_{k L}}{\partial z_{L}} \gamma_{k i} \alpha_{k L}(i), \\
f_{H} \frac{\partial v_{H}}{\partial z_{H}}=-\lambda \pi_{H}-\lambda \pi_{H} \sum_{j=1,2,3} \tau_{j} \frac{\partial c_{j H}}{\partial z_{H}}-\mu \frac{\partial v_{H}}{\partial z_{H}}+\pi_{H} \sum_{k=1,2} \sum_{i=L, H} \frac{\partial c_{k H}}{\partial z_{H}} \gamma_{k i} \alpha_{k H}(i) .
\end{gathered}
$$


The first-order condition with respect to commodity taxes $\tau_{j}, j=1,2,3$, read

$$
\begin{aligned}
& \sum_{i=L, H} f_{i} \frac{\partial v_{i}}{\partial \tau_{j}}+\lambda \sum_{i=L, H} \pi_{i} c_{j i}+\lambda \sum_{i=L, H} \sum_{n=1,2,3} \pi_{i} \tau_{n} \frac{\partial c_{n i}}{\partial \tau_{j}}+\mu\left(\frac{\partial v_{H}}{\partial \tau_{j}}-\frac{\partial v_{H}[L]}{\partial \tau_{j}}\right) \\
& -\sum_{k=1,2} \sum_{i=L, H} \gamma_{k L} \alpha_{k i}(L) \pi_{i} \frac{\partial c_{k i}}{\partial \tau_{j}}-\sum_{k=1,2} \sum_{i=L, H} \gamma_{k H} \alpha_{k i}(H) \pi_{i} \frac{\partial c_{k i}}{\partial \tau_{j}}=0
\end{aligned}
$$

Finally, first order conditions for $\overline{c_{k L}}$ and $\overline{c_{k H}}, k=1,2$, are given by:

$$
\begin{gathered}
f_{L} \frac{\partial v_{L}}{\partial \overline{c_{k L}}}+\lambda \pi_{L} \sum_{j=1,2,3} \tau_{j} \frac{\partial c_{j L}}{\partial \overline{c_{k L}}}-\mu \frac{\partial v_{H}[L]}{\partial \overline{c_{k L}}}+\gamma_{k L}-\pi_{L} \sum_{m=1,2} \sum_{i=L, H} \frac{\partial c_{m L}}{\partial \overline{c_{k L}}} \gamma_{m i} \alpha_{m L}(i)=0 \\
f_{H} \frac{\partial v_{H}}{\partial \overline{c_{k H}}}+\lambda \pi_{H} \sum_{j=1,2,3} \tau_{j} \frac{\partial c_{j H}}{\partial \overline{c_{k H}}}+\mu \frac{\partial v_{H}}{\partial \overline{c_{k H}}}+\gamma_{k H}-\pi_{H} \sum_{m=1,2} \sum_{i=L, H} \frac{\partial c_{m H}}{\partial \overline{c_{k H}}} \gamma_{m i} \alpha_{m H}(i)=0 .
\end{gathered}
$$

\section{Derivation of $\gamma_{k L} / \lambda$ and $\gamma_{k H} / \lambda$}

Further, we make use of the Slutsky decomposition $\frac{\partial c_{j i}}{\partial \bar{c}_{k i}}=\frac{\partial c_{j i}^{c o m}}{\partial \bar{c} c_{k i}}-M W P_{k i} \frac{\partial c_{j i}}{\partial x_{i}}$, which can be derived as follows. Let the expenditure function of an individual $i$ be denoted by $E_{i}\left(p_{1}, p_{2}, p_{3}, z_{i}, \overline{c_{1 i}}, \overline{c_{2 i}}, u\right)$, which in equilibrium is equal to $x_{i}$. Now use $c_{j i}\left(p_{1}, p_{2}, p_{3}, E_{i}(\cdot), z_{i}, \overline{c_{1 i}}, \overline{c_{2 i}}\right)=c_{j i}^{c o m}\left(p_{1}, p_{2}, p_{3}, z_{i}, \overline{c_{1 i}}, \overline{c_{2 i}}, u\right)$ and differentiate it with respect to $\overline{c_{k i}}$ to get $\frac{\partial c_{j i}}{\partial \bar{c}_{k i}}+\frac{\partial c_{j i}}{\partial x_{i}} \frac{\partial E_{i}}{\partial \overline{c_{k i}}}=\frac{\partial c_{j i}^{c o m}}{\partial \bar{c}_{k i}}$. Take the expenditure minimization problem of an individual $i$ and make use of the Envelope Theorem to get $\frac{\partial E_{i}}{\partial \bar{c}_{k i}}=M W P_{k i}$. All together this yields the Slutsky decomposition as written above.

Now take the first-order condition for $\overline{c_{k L}}$ given by (34) and add and subtract $\mu \frac{\partial v_{H}[L]}{\partial x_{L}} \frac{\partial v_{L} / \partial \overline{c_{k L}}}{\partial v_{L} / \partial x_{L}}$. Then (34) can be written as

$$
\begin{aligned}
& \left(f_{L} \frac{\partial v_{L}}{\partial x_{L}}-\mu \frac{\partial v_{H}[L]}{\partial x_{L}}\right) \frac{\partial v_{L} / \partial \overline{c_{k L}}}{\partial v_{L} / \partial x_{L}}-\mu \frac{\partial v_{H}[L]}{\partial x_{L}}\left(\frac{\partial v_{H}[L] / \partial \overline{c_{k L}}}{\partial v_{H}[L] / \partial x_{L}}-\frac{\partial v_{L} / \partial \overline{c_{k L}}}{\partial v_{L} / \partial x_{L}}\right)+\lambda \pi_{L} \sum_{j=1,2,3} \tau_{j} \frac{\partial c_{j L}}{\partial \overline{c_{k L}}} \\
& +\gamma_{k L}-\pi_{L} \frac{\partial c_{1 L}}{\partial \overline{c_{k L}}} \sum_{i} \gamma_{1 i} \alpha_{1 L}(i)-\pi_{L} \frac{\partial c_{2 L}}{\partial \overline{c_{k L}}} \sum_{i} \gamma_{2 i} \alpha_{2 L}(i)=0
\end{aligned}
$$

$k=1,2$. Make use of the definition for $M W P_{k i}$ and substitute for $\left(f_{L} \frac{\partial v_{L}}{\partial x_{L}}-\mu \frac{\partial v_{H}[L]}{\partial x_{L}}\right)$ from the first-order condition for $x_{L}$ given by (29). In addition use the Slutsky decom- 
positions $\frac{\partial c_{j i}}{\partial \overline{c_{k i}}}=\frac{\partial c_{j i}^{c o m}}{\partial \overline{c_{k i}}}-M W P_{k i} \frac{\partial c_{j i}}{\partial x_{i}}, j=1,2,3$ and $k=1,2$. Then one gets

$$
\begin{aligned}
\frac{\gamma_{k L}}{\lambda}= & \frac{1}{1-\alpha_{k L}(L) \pi_{L} \frac{\partial c_{k L}^{c o m}}{\partial \bar{c}_{k L}}}\left(\pi_{L} M W P_{k L}-\mu \frac{\partial v_{H}[L]}{\partial x_{L}}\left(M W P_{k H}[L]-M W P_{k L}\right)-\pi_{L} \sum_{j=1}^{3} \tau_{j} \frac{\partial c_{j L}^{c o m}}{\partial \overline{c_{k L}}}\right. \\
& \left.+\frac{\gamma_{m L}}{\lambda} \alpha_{m L}(L) \pi_{L} \frac{\partial c_{m L}^{c o m}}{\partial \overline{c_{k L}}}+\sum_{m=1,2} \frac{\gamma_{m H}}{\lambda} \alpha_{m L}(H) \pi_{L} \frac{\partial c_{m L}^{c o m}}{\partial \overline{c_{k L}}}\right),
\end{aligned}
$$

$k, m=1,2$ and $k \neq m$.

To get an expression for $\gamma_{k H} / \lambda$ take the first-order condition for $\overline{c_{k H}}$ (equation (35)) which can be converted to

$$
\begin{aligned}
& \left(f_{H} \frac{\partial v_{H}}{\partial x_{H}}+\mu \frac{\partial v_{H}}{\partial x_{H}}\right) \frac{\partial v_{H} / \partial \overline{c_{k H}}}{\partial v_{H} / \partial x_{H}}+\lambda \pi_{H} \sum_{j=1,2,3} \tau_{j} \frac{\partial c_{j H}}{\partial \overline{c_{k H}}}+\gamma_{k H}-\pi_{H} \frac{\partial c_{1 H}}{\partial \overline{c_{k H}}} \sum_{i} \gamma_{1 i} \alpha_{1 H}(i) \\
& -\pi_{H} \frac{\partial c_{2 H}}{\partial \overline{c_{k H}}} \sum_{i} \gamma_{2 i} \alpha_{2 H}(i)=0
\end{aligned}
$$

$k=1,2$. Substituting for $\left(f_{H} \frac{\partial v_{H}}{\partial x_{H}}+\mu \frac{\partial v_{H}}{\partial x_{H}}\right)$ from (30) and again making use of the Slutsky decompositions, (38) can be written as

$$
\begin{aligned}
\frac{\gamma_{k H}}{\lambda}= & \frac{1}{1-\alpha_{k H}(H) \pi_{H} \frac{\partial c_{k H}^{c o m}}{\partial \bar{c}_{k H}}}\left(\pi_{H} M W P_{k H}-\pi_{H} \sum_{j=1}^{3} \tau_{j} \frac{\partial c_{j H}^{c o m}}{\partial \overline{c_{k H}}}+\frac{\gamma_{m H}}{\lambda} \alpha_{m H}(H) \pi_{H} \frac{\partial c_{m H}^{c o m}}{\partial \overline{c_{k H}}}\right. \\
& \left.+\sum_{m=1,2} \frac{\gamma_{m L}}{\lambda} \alpha_{m H}(L) \pi_{H} \frac{\partial c_{m H}^{c o m}}{\partial \overline{c_{k H}}}\right),
\end{aligned}
$$

$k, m=1,2$ and $k \neq m$.

\section{Derivation of optimal commodity tax rates}

Take the first-order condition for $\tau_{j}$ given by (33) and plug in for $\frac{\partial v_{i}}{\partial \tau_{j}}=-c_{j i} \frac{\partial v_{i}}{\partial x_{i}}$, $\frac{\partial v_{H}[L]}{\partial \tau_{j}}=-c_{j H}[L] \frac{\partial v_{H}[L]}{\partial x_{L}}$ and for the Slutsky-equations $\frac{\partial c_{j i}}{\partial \tau_{j}}=\frac{\partial c_{j i}^{c o m}}{\partial p_{j}}-c_{j i} \frac{\partial c_{j i}}{\partial x_{i}}$ and $\frac{\partial c_{m i}}{\partial \tau_{j}}=$ $\frac{\partial c_{m i}^{c o m}}{\partial p_{j}}-c_{j i} \frac{\partial c_{m i}}{\partial x_{i}}$ with $j, m=1,2,3$ and $j \neq m$. One gets

$$
\begin{aligned}
& -c_{j L} f_{L} \frac{\partial v_{L}}{\partial x_{L}}-c_{j H} f_{H} \frac{\partial v_{H}}{\partial x_{H}}+\lambda \sum_{i} \pi_{i} c_{j i}+\lambda \sum_{m=1,2,3} \sum_{i} \tau_{m} \pi_{i}\left(\frac{\partial c_{m i}^{c o m}}{\partial p_{j}}-c_{j i} \frac{\partial c_{m i}}{\partial x_{i}}\right) \\
& -\mu\left(\frac{\partial v_{H}}{\partial x_{H}} c_{j H}-\frac{\partial v_{H}[L]}{\partial x_{L}} c_{j H}[L]\right)-\gamma_{1 L} \sum_{i} \alpha_{1 i}(L) \pi_{i}\left(\frac{\partial c_{1 i}^{c o m}}{\partial p_{j}}-c_{j i} \frac{\partial c_{1 i}}{\partial x_{i}}\right) \\
& -\gamma_{1 H} \sum_{i} \alpha_{1 i}(H) \pi_{i}\left(\frac{\partial c_{1 i}^{c o m}}{\partial p_{j}}-c_{j i} \frac{\partial c_{1 i}}{\partial x_{i}}\right)-\gamma_{2 L} \sum_{i} \alpha_{2 i}(L) \pi_{i}\left(\frac{\partial c_{2 i}^{c o m}}{\partial p_{j}}-c_{j i} \frac{\partial c_{2 i}}{\partial x_{i}}\right) \\
& -\gamma_{2 H} \sum_{i} \alpha_{2 i}(H) \pi_{i}\left(\frac{\partial c_{2 i}^{c o m}}{\partial p_{j}}-c_{j i} \frac{\partial c_{2 i}}{\partial x_{i}}\right)=0 .
\end{aligned}
$$


Substituting for $f_{L} \frac{\partial v_{L}}{\partial x_{L}}$ and $f_{H} \frac{\partial v_{H}}{\partial x_{H}}$ from (29) and (30), equation (40) can be written as

$$
\begin{gathered}
\sum_{m=1,2,3} \sum_{i} \tau_{m} \pi_{i} \frac{\partial c_{m i}^{c o m}}{\partial p_{j}}=-\frac{\mu}{\lambda} \frac{\partial v_{H}[L]}{\partial x_{L}}\left(c_{j H}[L]-c_{j L}\right)+\frac{\gamma_{1 L}}{\lambda} \sum_{i} \alpha_{1 i}(L) \pi_{i} \frac{\partial c_{1 i}^{c o m}}{\partial p_{j}} \\
+\frac{\gamma_{1 H}}{\lambda} \sum_{i} \alpha_{1 i}(H) \pi_{i} \frac{\partial c_{1 i}^{c o m}}{\partial p_{j}}+\frac{\gamma_{2 L}}{\lambda} \sum_{i} \alpha_{2 i}(L) \pi_{i} \frac{\partial c_{2 i}^{c o m}}{\partial p_{j}}+\frac{\gamma_{2 H}}{\lambda} \sum_{i} \alpha_{2 i}(H) \pi_{i} \frac{\partial c_{2 i}^{c o m}}{\partial p_{j}}
\end{gathered}
$$

$j=1,2,3$. There are three equations with three unknowns $\tau_{1}, \tau_{2}, \tau_{3}$. Thus, equation (41) can be transformed to matrix notation as given by (9) in the text.

\section{Derivation of optimal marginal income tax rates}

To get an expression for the optimal marginal income tax rate of an $H$-type divide the first order condition for $z_{H}$ and $x_{H}((32)$ by (30)) to get

$$
\frac{\left(f_{H}+\mu\right) \frac{\partial v_{H}}{\partial z_{H}}}{\left(f_{H}+\mu\right) \frac{\partial v_{H}}{\partial x_{H}}}=\frac{-\lambda \pi_{H}-\lambda \pi_{H} \sum_{j} \tau_{j} \frac{\partial c_{j H}}{\partial z_{H}}+\pi_{H} \frac{\partial c_{1 H}}{\partial z_{H}} \sum_{i} \gamma_{1 i} \alpha_{1 H}(i)+\pi_{H} \frac{\partial c_{2 H}}{\partial z_{H}} \sum_{i} \gamma_{2 i} \alpha_{2 H}(i)}{\lambda \pi_{H}-\lambda \pi_{H} \sum_{j} \tau_{j} \frac{\partial c_{j H}}{\partial x_{H}}+\pi_{H} \frac{\partial c_{1 H}}{\partial x_{H}} \sum_{i} \gamma_{1 i} \alpha_{1 H}(i)+\pi_{H} \frac{\partial c_{2 H}}{\partial x_{H}} \sum_{i} \gamma_{2 i} \alpha_{2 H}(i)} .
$$

Use the definition $M R S_{z x}^{i} \equiv-\frac{\partial v_{i} / \partial z_{i}}{\partial v_{i} / \partial x_{i}}$ and rewrite (42) as

$$
\begin{aligned}
& -M R S_{z x}^{H}\left(\lambda \pi_{H}-\lambda \pi_{H} \sum_{j} \tau_{j} \frac{\partial c_{j H}}{\partial x_{H}}+\pi_{H} \frac{\partial c_{1 H}}{\partial x_{H}} \sum_{i} \gamma_{1 i} \alpha_{1 H}(i)+\pi_{H} \frac{\partial c_{2 H}}{\partial x_{H}} \sum_{i} \gamma_{2 i} \alpha_{2 H}(i)\right)= \\
& -\lambda \pi_{H}-\lambda \pi_{H} \sum_{j} \tau_{j} \frac{\partial c_{j H}}{\partial z_{H}}+\pi_{H} \frac{\partial c_{1 H}}{\partial z_{H}} \sum_{i} \gamma_{1 i} \alpha_{1 H}(i)+\pi_{H} \frac{\partial c_{2 H}}{\partial z_{H}} \sum_{i} \gamma_{2 i} \alpha_{2 H}(i)
\end{aligned}
$$

After dividing by $\lambda \pi_{H}$, (43) can be rewritten as

$$
\begin{aligned}
1-M R S_{z x}^{H}= & \sum_{k=1,2}\left(\frac{\gamma_{k L}}{\lambda} \alpha_{k H}(L)+\frac{\gamma_{k H}}{\lambda} \alpha_{k H}(H)-\tau_{k}\right)\left(\frac{\partial c_{k H}}{\partial z_{H}}+M R S_{z x}^{H} \frac{\partial c_{k H}}{\partial x_{H}}\right) \\
& -\tau_{3}\left(\frac{\partial c_{3 H}}{\partial z_{H}}+M R S_{z x}^{H} \frac{\partial c_{3 H}}{\partial x_{H}}\right) .
\end{aligned}
$$

Using equation (5) yields equation (13) from the text. To arrive at the optimal marginal income tax for the $L$-types, first divide (31) by (29) to get

$$
\frac{f_{L} \frac{\partial v_{L}}{\partial z_{L}}}{f_{L} \frac{\partial v_{L}}{\partial x_{L}}}=\frac{-\lambda \pi_{L}-\lambda \pi_{L} \sum_{j} \tau_{j} \frac{\partial c_{j L}}{\partial z_{L}}+\mu \frac{\partial v_{H}[L]}{\partial z_{L}}+\pi_{L} \frac{\partial c_{1 L}}{\partial z_{L}} \sum_{i} \gamma_{1 i} \alpha_{1 L}(i)+\pi_{L} \frac{\partial c_{2 L}}{\partial z_{L}} \sum_{i} \gamma_{2 i} \alpha_{2 L}(i)}{\lambda \pi_{L}-\lambda \pi_{L} \sum_{j} \tau_{j} \frac{\partial c_{j L}}{\partial x_{L}}+\mu \frac{\partial v_{H}[L]}{\partial x_{L}}+\pi_{L} \frac{\partial c_{1 L}}{\partial x_{L}} \sum_{i} \gamma_{1 i} \alpha_{1 L}(i)+\pi_{L} \frac{\partial c_{2 L}}{\partial x_{L}} \sum_{i} \gamma_{2 i} \alpha_{2 L}(i)} .
$$

Again use the definition for $M R S_{z x}^{L}$ from above and divide (45) by $\lambda \pi_{L}$ and $\partial v_{H}[L] / \partial x_{L}$. 
Then (45) can be rewritten as

$$
\begin{aligned}
& -\frac{M R S_{z x}^{L}}{\partial v_{H}[L] / \partial x_{L}}\left(1-\sum_{j} \tau_{j} \frac{\partial c_{j L}}{\partial x_{L}}+\frac{\mu}{\lambda \pi_{L}} \frac{\partial v_{H}[L]}{\partial x_{L}}+\frac{\partial c_{1 L}}{\partial x_{L}} \sum_{i} \frac{\gamma_{1 i}}{\lambda} \alpha_{1 L}(i)+\frac{\partial c_{2 L}}{\partial x_{L}} \sum_{i} \frac{\gamma_{2 i}}{\lambda} \alpha_{2 L}(i)\right)= \\
& \frac{1}{\partial v_{H}[L] / \partial x_{L}}\left(-1-\sum_{j} \tau_{j} \frac{\partial c_{j L}}{\partial z_{L}}+\frac{\partial c_{1 L}}{\partial z_{L}} \sum_{i} \frac{\gamma_{1 i}}{\lambda} \alpha_{1 L}(i)+\frac{\partial c_{2 L}}{\partial z_{L}} \sum_{i} \frac{\gamma_{2 i}}{\lambda} \alpha_{2 L}(i)\right)-\frac{\mu}{\lambda \pi_{L}} M R S_{z x}^{H}[L] .
\end{aligned}
$$

Multiply (46) by $\partial v_{H}[L] / \partial x_{L}$ and use equation (5) to get equation (14) from the text.

\section{Acknowledgements}

I thank Johann K. Brunner, Susanne Pech and two anonymous referees for valuable comments and suggestions. This research was supported by the Austrian Science Funds (FWF).

\section{References}

ALPIZAR, F., F. CARLSSON, and O. JOHANSSON-STENMAN (2005) How much do we care about absolute versus relative income and consumption?, Journal of Economic Behavior \& Organization 56, 405-421.

ARISTOTLE (1924) Rhetoric, Book II, in: W. D. Ross (ed.), Works of Aristotle, Vol. XI, Oxford: Clarendon Press.

ARONSSON, T., and O. JOHANSSON-STENMAN (2008) When the Joneses' consumption hurts: optimal public good provision and nonlinear income taxation, Journal of Public Economics 92, 986-997.

ARONSSON, T., and O. JOHANSSON-STENMAN (2010) Positional concerns in an OLG model: optimal labor and capital income taxation, International Economic Review 51, 1071-1095.

ARONSSON, T., and O. JOHANSSON-STENMAN (2012) Conspicuous leisure: optimal income taxation when both relative consumption and relative leisure matter, Scandinavian Journal of Economics, forthcoming.

ATKINSON, A. B., and J. E. STIGLITZ (1976) The design of tax structure: direct versus indirect taxation, Journal of Public Economics 6, 55-75.

BOSKIN, M. J., and E. SHESHINSKI (1978) Optimal redistributive taxation when individual welfare depends upon relative income, Quarterly Journal of Economics 92, 589-601.

BOVENBERG, A. L., and L. H. GOULDER (2002) Environmental taxation and regulation, in: A. J. Auerbach and M. Feldstein (eds.), Handbook of Public Economics, vol. 3, North-Holland, Amsterdam, 1471-1545. 
BOWLES, S., and Y.-J. PARK (2005) Inequality, emulation, and work hours: was Thorsten Veblen right?, Economic Journal 15, 397-413.

CLARK, A. E., P. FRIJTERS, and M. A. SHIELDS (2008) Relative income, happiness and utility: an explanation for the Easterlin paradox and other puzzles, Journal of Economic Literature 46, 95-144.

CREMER, H., F. GAHVARI, and N. LADOUX (1998) Externalities and optimal taxation, Journal of Public Economics 70, 343-365.

CORNEO, G., and O. JEANNE (2001) Status, the distribution of wealth, and growth, Scandinavian Journal of Economics 103, 283-293.

DUPOR, B., and W. F. LIU (2003) Jealousy and equilibrium overconsumption, American Economic Review 93, 423-428.

DUESENBERRY, J. A. (1949) Income, saving and the theory of consumer behavior, Harvard University Press.

ECKERSTORFER, P. (2012) Optimal redistributive taxation in a multi-externality model, Finanzarchiv 68, forthcoming.

FRANK, R. (1999) Luxury Fever. Why money fails to satisfy in an era of excess, New York: Free Press.

FRANK, R. (2008) Should public policy respond to positional externalities?, Journal of Public Economics 92, 1777-1786.

IRELAND, N. J. (2001) Optimal income tax in the presence of status effects, Journal of Public Economics 81, 193-212.

KOPCZUK, W. (2003) A note on optimal taxation in the presence of externalities, Economics Letters 80, 81-86.

MEADE, W. (1952) External economies and diseconomies in a competitive situation, Economic Journal 62, 54-67.

MICHELETTO, L. (2008) Redistribution and optimal mixed taxation in the presence of consumption externalities, Journal of Public Economics 92, 2262-2274.

MICHELETTO, L. (2010) Optimal nonlinear redistributive taxation and public good provision in an economy with Veblen effects, Journal of Public Economic Theory 13, 71-96.

MIRRLEES, J.A. (1971) An exploration in the theory of optimal income taxation, Review of Economic Studies 38, 175-208.

NG, Y.-K. (1987) Relative income effects and the appropriate level of public expenditures, Oxford Economic Papers 39, 293-300.

OSWALD, A. J. (1983) Altruism, jealousy and the theory of optimal non-linear taxation, Journal of Public Economics 20, 77-87.

PIRTTILÄ, J., and M. TUOMALA (1997) Income tax, commodity tax and environmental policy, International Tax and Public Finance 4, 379-393. 
PLATO (2008) The Republic, Book II, Translated by B. Jowett, New York: Vintage Books.

RAE J. (1834) The new principle of political economy, in: Mixter, C. W. ed. (1905), The sociological theory of capital, New York: Macmillan.

SADKA, E. (1976) On income distribution, incentive effects and optimal income taxation, Review of Economic Studies 43, 261-268.

SANDMO, A. (1975) Optimal taxation in the presence of externalities, Swedish Journal of Economics 77, 86-98.

SEADE, J. (1977) On the shape of optimal tax schedules, Journal of Public Economics 7, 203-235.

SMITH A. (1759) The theory of moral sentiments, in: Raphael, D. D., and A. L. Macfie eds. (1976), The theory of moral sentiments, Oxford University Press.

SOLNICK, S., and D. HEMENWAY (1998) Is more always better? A survey on positional concerns, Journal of Economic Behavior \& Organization 37, 373-383.

SOLNICK, S., and D. HEMENWAY (2005) Are positional concerns stronger in some domains than in others, American Economic Review, Papers and Proceedings 45, 147151.

STIGLITZ, J. E. (1982) Self-selection and Pareto efficient taxation, Journal of Public Economics 17, 213-240.

VEBLEN, T. (1899) The theory of the leisure class, The Macmillan Company, New York.

WENDNER, R., and L. H. GOULDER (2007) Status effects, public good provision, and the excess burden, Journal of Public Economics 92, 1968-1985.

WENDNER, R. (2010) Growth and keeping up with the Jonses, Macroeconomic Dynamics 14, 176-199.

WENDNER, R. (2012) Ramsey, Pigou, heterogeneous agents, and non-atmospheric consumption externalities, Journal of Public Economic Theory 14, forthcoming. 